\title{
A relação entre cultura escolar e cultura jovem como geradores de oportunidades
}

\section{The relationship between school culture and youth culture as generators of opportunities}

\author{
Jefferson Riule, Ecleide Cunico Furlanetto \\ Universidade Cidade de São Paulo - Unicid
}

\begin{abstract}
Resumo
O objetivo desta pesquisa foi investigar as narrativas de alunos e docentes do Programa Aprendizagem Comercial do Senac SP a fim de identificar a relação entre cultura escolar e jovem como geradores de oportunidades. Estabeleceu diálogos teóricos sobre as culturas escolares e juvenis e de estudos sobre narrativas. As narrativas foram produzidas por dois grupos focais com seis alunos e com seis docentes. Ouvir os relatos de experiências possibilitou aprofundar a reflexão acerca da escola. Constatou-se que as culturas escolares e juvenis, favorecem o protagonismo juvenil, cria alternativas e práticas institucionais e educacionais que dialogam com o adolescente, criando oportunidades.

Palabras clave: educação profissional, cultura escolar, cultura juvenil, narrativas de profesores, narrativas de alunos.
\end{abstract}

\begin{abstract}
The objective of this research was to investigate the narratives of students and teachers of the Commercial Learning Program of Senac SP in order to identify the relationship between school culture and youth as generators of opportunities. He established theoretical dialogues on school and juvenile cultures and studies on narratives. The narratives were produced by two focus groups with six students and six teachers. Listening to the reports of experiences made it possible to deepen the reflection about the school. It was found that school and youth cultures favor youth protagonism, create institutional and educational alternatives and practices that dialogue with adolescents, creating opportunities.

Keywords: professional education, school culture, youth culture, teacher narratives, student narratives.
\end{abstract}

\section{Introdução}

Sou técnico de desenvolvimento profissional de uma instituição de ensino que atua e contribui há 70 anos, para a educação profissional oferecendo cursos formação inicial, técnicos e de ensino superior (graduação, extensão universitária e pós-graduação), presenciais ou a distância, em diferentes áreas do conhecimento.

Com uma trajetória longa no Senac, dos quais os últimos 10 anos, na equipe da Gerência de Operações 2 (GO2), cuja função é promover a articulação, o monitoramento e a distribuição de produtos e serviços educacionais do Senac São Paulo e, nessa perspectiva, o papel das GOs está diretamente relacionado ao processo de planejamento estratégico da oferta e ao seu acompanhamento na rede, sendo um suporte direto para as unidades alinharem suas ações cotidianas com as macro estratégias e diretrizes definidas pela organização.

Dentro da GO2, há 4 anos, componho o grupo de trabalho denominado "Frente Oferta", que tem por objetivo acompanhar metas corporativas, orientar a operação do Programa Aprendizagem Comercial, a composição dos percentuais de alunos do Programa Senac de Gratuidade (PSG) em sala de aula e apoio às atividades das equipes de Assistentes Sociais. Um dos principais objetivos desta Frente, é a gestão do PSG que promove o acesso à educação profissional de qualidade, por meio da Política Senac de Concessão de Bolsas de Estudo. O Programa Aprendizagem Comercial compõe a oferta do PSG e é onde se concentra o maior número de vagas de todas as unidades educacionais.

Acompanhando o Programa Aprendizagem Comercial, pude observar que, apesar da proposta procurar garantir condições de ensino e aprendizagem adequadas para docentes e alunos, existem docentes e alunos insatisfeitos e infelizes. Várias ações são realizadas com as unidades, a fim de entendermos as principais dificuldades operacionais. Além da avaliação institucional, instrumento que compila a opinião dos alunos e docentes, em 2015 e 2016 foram realizados encontros com técnicos, docentes coordenadores e docentes do Programa Aprendizagem. Os resultados dessas ações permitem um alinhamento estratégico, educacional e operacional sobre o programa, além de sensibilizar as equipes sobre temas relevantes, complexos e institucionais.

Além dos resultados acima destacados, com base na minha observação pude perceber que algumas atitudes por parte dos docentes provocam tensões que se manifestam nas relações entre eles e os alunos. A primeira que merece destaque é o preconceito, por parte da maioria dos docentes em relação ao perfil dos alunos do Programa. 
Outra a ser enfatizada é a falta de habilidade dos docentes em lidar com adolescentes, constatada por meio das posturas rígidas assumidas por docentes que, inclusive, propõe regras inflexíveis no que se refere a vestimentas e comportamentos a serem adotados, apenas para os alunos do Programa, diferentes das adotadas para os outros alunos do Senac, o que os discrimina em relação aos outros.

Essas constatações me levaram a revisar a literatura buscando no Banco de teses da CAPES e na SciELO Scientific Electronic Library Online, em novembro de 2015, publicações que tivessem como descritores: cultura escolar e cultura juvenil. Os resultados obtidos mostram que essas duas dimensões foram pouco relacionadas nas pesquisas, sobretudo, no que se refere à educação profissional.

Com base na minha trajetória de vida, que se assemelha a de muitos dos participantes do Programa Aprendizagem, jovens em situação de vulnerabilidade que desde cedo necessitam trabalhar, sustentar a família e estudar, apoiado, também, na minha experiência profissional e na revisão de literatura, propus como objetivo principal deste estudo:

Investigar as narrativas de alunos e docentes do Programa Aprendizagem Comercial do Senac SP no sentido de identificar a relação entre cultura escolar $e$ cultura jovem como geradores de oportunidades, no processo de socialização e aprendizagem que ocorre no espaço escolar.

Elencamos como objetivos específicos: Contextualizar o panorama institucional sobre a educação profissional e o Programa Aprendizagem Comercial; Dialogar com autores que investigam as narrativas de vida, cultura escolar, adulta e juvenil e analisar as narrativas dos alunos e docentes do Programa, no sentido de identificar oportunidades na vida dos jovens.

\section{Método}

Esta pesquisa apoiou-se na abordagem qualitativa (Stake, 2011), mais especificamente nos pressupostos da pesquisa narrativa de acordo com Bauer (2002), Galvão (2005), entre outros autores. Os sujeitos de pesquisa foram alunos e docentes do Programa Aprendizagem, tendo como procedimento de coleta de dados dois grupos focais segundo Gatti (2005) e Powell (1996).

É importante observar que as narrativas de vida, como procedimento de investigação qualitativa, na área de educação, procuram reconhecer as trajetórias de docentes e alunos, para que o exercício da aprendizagem, seja elaborado por meio da construção de subjetividades, relacionando ações educativas, cultura escolar e políticas educacionais. Dessa forma a pesquisa narrativa cumpre a função de revelar e legitimar experiências de diferentes atores sociais que ao narrá-las se reconhecem como sujeitos de suas histórias.

\section{Discussão}

\section{Juventude: uma Construção Social}

O Governo Federal, por meio do Portal Brasil, apresenta informações sobre a população mundial: hoje, uma em cada cinco pessoas tem entre 15 e 24 anos, e cerca de $85 \%$ desses jovens vive em países em desenvolvimento. Em âmbito nacional, o Censo de 2010, aponta que os jovens brasileiros entre 15 e 29 anos, representam $25 \%$ da população, formando um grupo extremamente heterogêneo.

A Secretaria Nacional da Juventude (SNJ) publicou uma pesquisa sobre o perfil da juventude no Brasil em que seis a cada dez jovens se declararam pretos (15\%) ou pardos (45\%). A ONU sinaliza que além da diversidade, a desigualdade é um componente de destaque entre esses jovens, intimamente influenciada pelo preconceito, pela discriminação.

Apresentar o conceito de juventude ou cultura jovem, principalmente no Brasil, não se trata de uma tarefa fácil segundo Costa (2000). O tema é complexo e há ampla bibliografia publicada.

Ter em mente essa complexidade, no entanto, é fundamental para evitar equívocos no uso desse conceito, principalmente quando se trata da formulação e da implementação de políticas públicas dirigidas a esse segmento da população (2000, p.66).

Segundo Costa (2000), o conceito de juventude irá variar conforme o interesse específico de quem o utiliza (cientistas políticos, educadores, médicos, publicitários), ou de acordo com seu contexto econômico, social, histórico e cultural, logo, se faz necessário localizar e datar as circunstancias que serão abordadas. Outro ponto relevante é a conceituação que o próprio jovem se faz de si.

O autor afirma que a maior parte das definições de juventude são mais voltada para a delimitação do fenômeno do que para a explicação das singularidades que ocorrem nessa fase da vida um exemplo disto, é o enfoque dado pelas Nações Unidas, em que a idade do indivíduo é que define sua fase. Do ponto de vista demográfico está proposição pode até ser favorável, no entanto, do ponto de vista jurídico abarca indivíduos titulares de status legais inteiramente distintos. Conforme afirmação: “A definição clássica de juventude como trânsito entre a infância e a idade adulta, por sua vez, parece preocupar-se mais com o que a juventude não é (infância e idade adulta) do que com aquilo que ela realmente é (Costa, 2000, p. 67)".

A criança e o adolescente gozam de direitos fundamentais inerentes à pessoa humana, sem prejuízo da proteção integral de que trata a lei. A Doutrina da proteção integral é defendida pela ONU com base na declaração universal dos direitos da criança e é um reflexo direto do que está em nossa Constituição Federal de 1988 nos seus artigos 227 e 228.

Essa falta de esclarecimento entre faixa etária do jovem e a transição da fase infantil para a vida adulta, provoca 
uma grande fenda e contradição no que se refere às responsabilidades físicas, psicológicas, sociais e legais e de cobrança da sociedade civil. Compreender o jovem em seu espaço, momento e características, se faz necessário para compreender sua maneira de pensar e agir, sobretudo, dentro do contexto escolar, em que a socialização com outros jovens, possibilita criar vínculos gerando uma subjetividade.

Rodriguez (2002) em seus estudos sobre a fase da adolescência no âmbito das ciências sociais, aponta três aspectos importantes da cultura jovem: A influência dos grupos de pares na socialização da juventude, principalmente como influentes da transmissão de "culturas" especificas, ou modismo de práticas especificas; ou ainda das chamadas "tribos urbanas" (grupos de canto, futebol). Por fim, uma terceira categoria chamada expressões culturais da juventude, totalmente ligadas a música e aspecto culturais.

Percebe-se que o jovem, nessa fase da vida, se junta a grupo de pares com os quais de alguma maneira se identifica, esses grupos assumem quase uma identidade coletiva que imprimindo marcas na subjetividade dos jovens nesta fase da vida. Por sua vez, os meios de comunicação de massa e produção cultural, aproveitam deste momento, para desenvolverem produtos, programas, personagens, a fim de criaram mensagens, desenvolverem uma dinâmica de consumo midiático, comportamental e de produto, impactando os modelos de grupo e o consumo propriamente dito.

A tecnologia da informação e comunicações, é outra parceira aliada do jovem. Ela avança rapidamente com seus recursos e inovações, embora, ainda sob a ótica de Rodriguez (2002), a cultura escolar, não acompanha esse movimento em suas práticas:

Mesmo quando a educação é decidida a usar a nova tecnologia da informação e comunicações, fá-lo a partir dos paradigmas existentes na própria cultura escolar, que produz programas excessivamente formalizados e totalmente chatos aos seus destinatários, eles reagem categoricamente, que institui o "veto" adequado, ou seja, a decisão de não participar em todas as suas mensagens e propostas (2002, p. 4).

Fanfani (2001) apresenta a adolescência e a juventude como construções sociais, classes de idades, que possuem a mesma base biológica e representações históricas relativamente arbitrárias. $\mathrm{O}$ autor salienta, por sua vez, que essas fronteiras são confusas e que esses limites sociais são sempre imprecisos e variáveis, no entanto, a maioridade, de forma variada, está claramente estabelecida nos códigos e nas leis de todas as sociedades. O próprio sistema educacional formal não é arbitrário, levando em conta que se ingressa na escola a partir de uma determinada idade. Nessa perspectiva, a própria escola, também, contribui para a construção social da juventude como um tempo de vida entre a infância e a condição de adulto, um tempo de preparação e de espera. Ser jovem é permanecer em um status incompleto, caracterizado por um período de formação física, intelectual e social, estabelecido entre a puberdade e a fase adulta com suas responsabilidades.

\section{A relação entre as Culturas Escolar e Jovem}

A fim de investigar as narrativas de alunos e docentes do Programa Aprendizagem Comercial do Senac SP, é que se inicia essa discussão sendo importante alinhar e conceituar temas que permeiam essas culturas e que emergem diariamente com a prática dentro do processo de escolarização e seus espaços.

Em meio a revisão de literatura para construção desta pesquisa, encontrou-se um artigo que teve como objetivo examinar o modo como a noção de cultura escolar vem sendo apropriado pelas pesquisas que tomam a escola brasileira como objeto de estudo. Nesse estudo, foram analisadas a produção de pesquisadores dos programas de pós-graduação stricto sensu entre os anos de 1987 e 2007 e com base nisso, foram localizados trezentos e sessenta e um (361) resumos de dissertações e teses, que faziam referência ao termo cultura escolar.

O estudo realizado pelas autoras Knoblauch et al. (2012), permite compreender que a escola tem uma cultura que se diferencia de outras instituições, em função de seus valores e seu sistema simbólico. Com base em Faria Filho et al. (2004 apud Knoblauch et al., 2012), as autoras destacam três grandes eixos que vêm norteando as pesquisas realizadas: saberes, conhecimentos e currículos; espaços, tempos e instituições escolares e materialidade escolar e métodos de ensino. Elas também apresentam algumas definições de diferentes autores que favorecem uma maior aproximação do conceito de cultura escolar.

A primeira delas é Julia (2001 apud Knoblauch et al., 2012) que se refere à cultura escolar como:

Um conjunto de normas que definem conhecimentos a ensinar e condutas a incultar, e um conjunto de práticas que permitem a transmissão desses conhecimentos e a incorporação desses comportamentos; normas e práticas coordenadas a finalidades que podem variar segundo épocas (2012, p. 4).

Viñao Frago (1993 apud Knoblauch et al., 2012) se aproxima de Julia (2001 apud Knoblauch et al., 2012) ao afirmar que a cultura escolar é um:

Conjunto de aspectos institucionalizados incluye prácticas y conductas, modos de vida, hábitos y ritos - la historia contidiana del hacer escolar -, objetivos materiales - función, uso, distribución em el espacio, materialidade física, simbologia, introduction, transformación, desaparición... y modos de pensar, así como significados e ideas compartidas. (2012, p. 68-69).

Forquim (1993 apud Knoblauch et al., 2012) com base na sociologia e na antropologia diferencia cultura escolar de cultura da escola.

A escola é também um "mundo social", que tem suas características de vida própria, seus ritmos e seus ritos, sua linguagem, seu imaginário, seus 
modos próprios de regulação e de transgressão, seu regime próprio de produção e de gestão de símbolos. E esta "cultura da escola" (no sentido em que se pode também falar da "cultura da oficina" ou da "cultura da prisão) não deve ser confundida tampouco com o que se entende por "cultura escolar", que se pode definir como um conjunto dos conteúdos cognitivos e simbólicos que, selecionados, organizados, "normalizados", "rotinizados", sob os efeitos dos imperativos de didatização, constituem habitualmente o objeto de uma transmissão deliberada no contexto das escolas (2012, p. 561).

Knoblauch et al. (2012) constataram que 86,70\% da produção de dissertação pesquisada, ou seja, trezentos e um (301) textos utiliza o termo cultura escolar, no entanto, com base na análise percebeu-se que a maioria faz uso desse termo de forma variada, ou como forma de caracterizar a dinâmica escolar.

Silva (2006 apud Knoblauch et al., 2012) confirma que:

Seja cultura escolar ou cultura da escola, esses conceitos acabam evidenciando praticamente a mesma coisa, isto é, a escola é uma instituição da sociedade, que possui suas próprias formas de ação e de razão, construídas no decorrer da sua história, tomando por base os confrontos e conflitos oriundos do choque entre as determinações externas a ela e as suas tradições. (2012, p. 7).

Silva (2006) também concorda com estudo de Knoblauch et al. (2012) enfatizando que toda escola possui uma cultura e uma identidade que é composta pela sua estrutura e pelos relacionamentos entre as pessoas, logo, entender a escola como um espaço de socialização e convergências de culturas nos faz caminhar para um conceito de cultura de escola ou cultura escolar que envolve todos os processos de interação, práticas e políticas, conforme define Silva:

A escola é uma instituição da sociedade, que possui suas próprias formas de ação e de razão, construídas no decorrer da sua história, tomando por base os confrontos e conflitos oriundos do choque entre as determinações externas a ela e as suas tradições, as quais se refletem na sua organização e gestão, nas suas práticas mais elementares e cotidianas, nas salas de aula e nos pátios e corredores, em todo e qualquer tempo, segmentado, fracionado ou não (SILVA, 2006, p. 28).

Sob a ótica das ciências sociais, Péres Gómes (2001 apud Knoblauch et al., 2012) considera a escola como um cruzamento de culturas de diferentes propostas, ou seja, a cultura crítica (produção cultural acumulada ao longo da história humana), a cultura acadêmica (localizada no currículo), a cultura social (valores hegemônicos), a cultura institucional da escola (normas, rituais, rotinas), e pôr fim a cultura da experiência do aluno, obtida em sua vida diária.

Esses cruzamentos entre culturas dentro de espaço escolar impactam diretamente com outra cultura a do jovem que também faz parte da escola. Nessa perspectiva Fanfani, (2001) reflete sobre o que oferece a educação escolar aos jovens da América Latina e em que medida, o que se oferece, atende às necessidades e expectativas das novas gerações de jovens latinos. Ao fazer isso, destaca um fenômeno importante que contribuiu para a introdução de uma nova categoria de jovens na escola, a massificação da escola, afetando o crescimento quantitativo não acompanhado por um aumento proporcional em recursos públicos investidos no setor da educação e contribuíram para a construção de novos sujeitos sociais nos espaços escolares produzindo conforme o autor:

Uma série de transformações nas instituições escolares. A velha escola secundária reservada às elites deve hoje responder à demanda de novos contingentes de postulantes. E, por força das circunstâncias, as mudanças não podem acarretar prejuízos de qualidade. As instituições, como sistemas de regras e recursos que estruturam as práticas sociais e educativas, mudam de forma e significado. Os velhos dispositivos que regulavam a relação professor-aluno e a relação com o conhecimento, que garantiam a autoridade pedagógica e produziam uma ordem institucional, se corroem quando deixam de ser eficientes e significativas na vida dos atores envolvidos. (Fanfani, 2001, p. 01).

Nesse sentido, Ribeiro (2014) complementa que devido a massificação escolar, a justiça na escola passou a ser vivenciada como tragédia, pois trouxe para seu interior princípios que provocam conflitos sociais, transformando, dessa forma a percepção do que é considerado justo ou injusto na de educação escolar, a promessa de que a escola garantiria igualdade de oportunidades não pode ser cumprida, na medida em que não elimina a correlação entre desigualdades sociais, desempenho escolar e inserção social dos indivíduos.

Fanfani, (2001) afirma que em função desses fenômenos a morfologia social dos alunos modificou significativamente. Os adolescentes e jovens atuais, são diferentes, dos primeiros "clientes" da educação escolar moderna. Nesse sentido:

Todas estas transformações na demografia, na morfologia e a cultura das novas gerações põem em crise a oferta tradicional de educação escolar. Os sintomas mais evidentes e estridentes são a exclusão e o fracasso escolar, o mal-estar, o conflito e a desordem, a violência e as dificuldades de integração nas instituições e, sobretudo, a ausência de sentido da experiência escolar para uma porção significativa de adolescentes e jovens latino-americanos (em especial aqueles que provêm de grupos sociais excluídos e subordinados) que têm dificuldades 
para ingressar, progredir e se desenvolver em instituições que não foram feitas para eles. (2001, p. 02)

De acordo com Ribeiro (2014, p. 4) com base em Bourdieu e Passeron, diz que:

Os autores afirmaram que a maior parte dos alunos provenientes das classes sociais mais desprovidas passava pela escola com uma vida escolar mais conturbada por reprovações e evasões e quando conseguia chegar à universidade, vinculava-se a cursos menos valorizados socialmente.

O processo de escolarização torna-se obrigatório, no entanto, no bojo desse processo de massificação, a instituição escolar perdeu sua capacidade de impor regras que determinem essa questão. O Estado, responsável por garantir o cumprimento da obrigatoriedade, se vê forçado a ampliar a oferta escolar cobrindo as condições mínimas, criando programas de bolsas de estudos, subsidiando e apoiando os jovens e suas famílias.

Se antes tínhamos a desigualdade social, com base em Ribeiro (2014) podemos dizer que passamos a ter "desigualdade escolar", relacionada ao princípio de justiça nas sociedades democráticas que demarcam as diferenças de distribuição de bens entre pessoas ou grupos de pessoas (ou classes sociais) que acabam por afetar o resultado escolar. A desigualdade escolar por sua vez gera o fracasso escolar. Louzano, (2013), o considera um fenômeno complexo relacionado às oportunidades educacionais desiguais que afeta de forma diferente alunos de distintos grupos econômicos, sociais e étnicoraciais.

A questão da desigualdade e exclusão escolar, existentes hoje não são mais as mesmas e nem ocorrem nos mesmos termos da que ocorriam no passado, conforme Oliveira (2007, p. 682):

Setores mais pobres reprovam mais, evadem mais, concluem menos, o mesmo ocorre com negros e meninos, mas, mais importante que isso, aprovam mais, permanecem mais e concluem mais do que em qualquer outro momento de nossa história educacional, ainda que permaneçam como os setores mais excluídos. Só que não são excluídos da mesma maneira que no passado!

A questão principal que Oliveira coloca é olhar para a positividade do processo de escolarização, os desafios ainda estão presentes, mas que há conquistas, perspectivas e principalmente oportunidades para esses jovens se incluírem mais, evadirem menos, concluírem mais e trabalharem mais.

E nesse sentido é importante salientar que as políticas públicas devem encarar como um dos seus desafios principais, desenvolver programas, que acolham esse perfil de jovem cujo intuito seja despertar o espirito protagonista, inovador e empreendedor, visando abrir novas oportunidades de desenvolvimento, aprendizagem profissional e carreira para esses jovens. Essas podem ser consideradas marcas formativas de programas que dialogam diretamente com o mundo do trabalho que buscam promover mudanças na vida desses adolescentes e de suas famílias, transformando, criando possibilidades para esses alunos escapem do estigma de alunos fracassados, lutando dessa forma pela mudança e diminuição da desigualdade escolar, ao favorecer políticas que abram oportunidades para todos os alunos.

Nessa perspectiva surgem no contexto educacional programas que tentam abrir as portas para esses jovens, buscando desenvolver ações educativas que motivem e potencializem as suas capacidades e competências, que inovem na metodologia e, que se regularizem de maneira democrática com base em regras construídos conjuntamente, e que sobretudo, valorize a condição de origem e vida social desses jovens.

\section{Resultados}

\section{Gerando oportunidades!}

Ao iniciar este estudo e com base na minha experiência e observação profissional, pude notar regras da cultura escolar e algumas atitudes por parte dos docentes que provocam tensões entre eles e os alunos. Um certo preconceito, falta de habilidade dos docentes em lidar com o perfil e a cultura jovem, posturas rígidas, regras inflexíveis, principalmente no que se refere a vestimentas e comportamentos a serem adotados apenas para os alunos do Programa Aprendizagem, o que para mim, os discriminava em relação aos demais alunos. Essa percepção inicial não se materializou, pois os dados de pesquisa nos mostraram dimensões diferentes, o que me levou a refazer, reconstruir a minha percepção e parâmetros e a constatar que não há impactos ou perdas no processo educacional.

Há tensões que surgem em função da própria rotina da comunidade escolar e suas questões administrativas, outras estabelecidas na construção das relações diárias entre ambos sujeitos de pesquisa e o ambiente escolar. Por fim, talvez uma questão "social", no olhar deste pesquisador, não ficando clara, mas parece pairar como se fosse uma "nuvem" sob o Programa Aprendizagem. No entanto, ora mais tensos nas falas dos docentes, ora mais leve nas vozes dos alunos, absolutamente nada interferem no processo de aprendizagem e formação de ambos os sujeitos, sobretudo, para os alunos que reconhecem uma transformação em suas vidas e valorizam a relação com o grupo de docentes e sua passagem pelo Programa Aprendizagem Comercial, como uma grande oportunidade de mudança de vida.

Retomando o objetivo desta pesquisa, foi possível entender que a maior tensão é provocada pelo esforço da instituição Senac SP, no sentido de criar um ritual de passagem da cultura juvenil para uma cultura adulta. Todos aqueles símbolos que representam a cultura juvenil, vão sendo, de uma certa forma, "esvaziados", substituídos, e existe uma busca dos docentes, no sentido dos alunos irem assumindo uma representação do adulto trabalhador, ou seja, passar pelo programa não significa apenas aprender questões técnicas e de conteúdo, mas significa também passar por um processo de transformação. 
Foi realizado um levantamento bibliográfico com o objetivo de identificar autores que dialogassem com os temas Educação Profissional no Brasil, Cultura Jovem e Cultura Escolar. Este estudo permitiu compreender, mais acerca da "Educação Profissional no Brasil", sua trajetória histórica, até os dias atuais e principalmente sua contribuição política e social.

Possibilitou entender os conceitos da cultura juvenil e escolar, relacionando-as com massificação escolar, estabelecendo o perfil dos jovens que ocupam as salas de aula atualmente, sua origem e seus anseios de vida, favorecendo a compreensão da importância que a escola tem como mobilizadora de práticas educacionais interessantes para esses jovens, capazes de mostrar o valor e o significado que aprender pode ter na vida deles.

Foi possível, também, se aprofundar no conceito de histórias de vida como metodologia de pesquisa qualitativa na área da educação e a organizar dois grupos focais, com o intuito de produzir narrativas que auxiliassem a reflexão sobre a atividade pedagógica num programa de educação profissional, em que os sujeitos de pesquisa, alunos e docentes, pudessem relatar as suas relações e vivências dentro do espaço educacional.

Esta pesquisa possibilitou constatar que a profissionalização e a inserção do aluno no mercado de trabalho é algo que a escola tradicional não está dando conta e, nesse sentido, surgem programas que buscam compensar essa lacuna deixada por esse sistema educacional, como o Programa Aprendizagem Comercial, que nasce no momento histórico em que a escola, não responde adequadamente às necessidades de grande parte dos jovens, no que se refere à inserção no mundo profissional. Existe uma distância muito grande entre o que se aprende na escola e o que é exigido no mundo profissional. O Programa Aprendizagem surge, neste intervalo, para preencher o vazio que está posto socialmente. No atual momento, em que pensam na reforma do ensino médio, talvez, programas como este tenham contribuições importantes, na medida em que eles estão atendendo às expectativas dos jovens que querem ter uma oportunidade de inserção no mundo do trabalho.

Esse jovem vê no programa uma oportunidade, mesmo que implique sacrificar sua antiga identidade, de ganhar autonomia, liberdade e, sobretudo, se sentir valorizado e reconhecido. Para as camadas mais vulneráveis, ter um emprego não significa apenas ganhar algum dinheiro, mas, sim, conquistar um lugar social.

Uma das situações mais difíceis nesse processo, talvez, seja sair de uma determinada classe social. Para isso, o sujeito precisa se autorizar fazer essa passagem, ter alguém que, de certa forma, tenha vivido esse processo e possa guiá-lo nessa caminhada se torna providencial. Nessa perspectiva, os alunos, talvez, valorizem o Programa Aprendizagem por constituir num espaço de positividade, em que encontram mentores capazes de acompanhá-los nessa empreitada que consiste em se introduzir no mundo do trabalho.

Além da oportunidade de emprego, os alunos do Programa Aprendizagem passam a almejar outras conquistas, entre elas, a possibilidade de continuarem seus estudos que consiste na entrada no Ensino Superior.
Ao passarem pelas empresas, nos rodízios de funções, eles percebem que quem dá continuidade aos estudos pode percorrer um caminho mais consistente do ponto de vista profissional.

Para finalizar, foi possível constatar que as culturas escolares e juvenis, neste contexto de pesquisa, são grandes geradoras de oportunidades, pois, contudo, favorecem o lugar do protagonismo dos jovens, criando alternativas e práticas institucionais e educacionais que dialoguem com o universo do adolescente, utilizando expressões, linguagens, comunicação e tecnologia que gerem sentidos, possibilitando as convergências entre as duas culturas e gerando transformações e oportunidades na vida desses jovens, garantindo e preservando a dignidade e a trajetória escolar dos alunos e de sua condição de origem.

\section{Referencias}

Amaral, M. (2006). Culturas juvenis X cultura escolar: repensando as noções de (in) disciplina $e$ autoridade no âmbito da educação. In: Proceedings of the 6th Psicanálise, Educação e Transmissão, [online] [cited 29 August 2016].

Azevedo, L.A., Shiroma, E.O. \& Coan, M. (2012). As políticas públicas para a educação profissional $e$ tecnológica: sucessivas reformas para atender a quem? RJ: B. Téc. Senac: a R. Educ. Prof.

Barbosa, M.S. (2014). Culturas escolares, culturas de infância e culturas familiares: As socializações e a escolarização no entretecer destas culturas. Campinas: vol. 28, n. 100 - Especial, p. 1059 - 1083. Disponível em: www.cedes.unicamp.br. Acesso em: 20 out. 2014.

Bauer, M.W., Gaskell, G. (2002) Pesquisa qualitativa com texto, imagem e som: um manual prático. RJ:Vozes. Brasil (2013). Ministério do Trabalho e Emprego. Portaria $n^{\circ} 1005$, de 01 de julho de 2013. Altera a Portaria $n^{\circ} 723$, de 23 de abril de 2012, que dispõe sobre o Cadastro Nacional de Aprendizagem Profissional - CNAP, destinado ao cadastramento das entidades qualificadas em formação técnicoprofissional

metódica. <http://www.normaslegais.com.br/legislac ao/portaria-mte-1005-2013.htm>

Brasil (2012). Portaria $n^{\circ}$ 723, de 23 de abril de 2012.

$<$ http://www.normaslegais.com.br/legislacao/portariamte-723-2012.htm>.

Brasil (2009). Ministério do Trabalho e Emprego. Portaria MTE $n^{\circ} 2.185$, de 5 de novembro de 2009. Disciplinar a oferta de cursos de aprendizagem profissional em nível de técnico de ensino. Disponível em:

$<$ http://acesso.mte.gov.br/data/files/8A7C816A32DC11 5D0132FEEF22115BB5/p_20091105_2185.pdf $>$.

Brasil (2016). Ministério do Trabalho e Emprego. Manual da Aprendizagem - MTE. $<$ http://portal.mte.gov.br/portal-mte/>

Costa, A.G. (2000). Protagonismo juvenil: adolescência, educação e participação democrática. Salvador: Fundação Odebrecht. 
Fanfani, E.T. (2001). Culturas jovens e cultura escolar. Documento apresentado no seminário "Escola Jovem: um novo olhar sobre o ensino médio”. Brasília: Organizado pelo Ministério da Educação. Secretaria de Educação Média e Tecnológica. Coordenação-Geral de Ensino Médio.

Galvão, C. (2005). Narrativas em Educação. Ciência \& Educação, v.11, n.2, p. 327-345.

Gatti, B.A. (2005). Grupo focal na pesquisa em ciências Sociais e Humanas. Brasília: Líber Livro Editora.

Gualtieri, R., Lugli, R. (2012). A escola e o fracasso escolar. São Paulo: Cortez.

Knoblauch, A. (2012). Levantamento de pesquisas sobre cultura escolar no Brasil. São Paulo: Educação e Pesquisa, v.38 n. 03, p. 557-574.

Louzano, P. (2013). Fracasso escolar: evolução das oportunidades educacionais de estudantes de diferentes grupos raciais. Cadernos Cenpec, v.3, n.1, p.111-133.

Oliveira, R.P. (2007). Da universalização do ensino fundamental ao desafio da qualidade: uma análise histórica. Campinas: Educação e Sociedade, v. 28, n. 100 - especial, p. 661-690.

Powell, R.A., Single, H.M. (1996). Focus groups. International Journal of Quality in Health Care, v.8, n.5, p. 499-504.

Ribeiro, V.M. (2014). Igualdade de Base: fundamentos de um princípio de justiça para políticas de Educação Básica. In: II Jornadas Latino americanas de Estudios Epistemológicos en Política Educativa. Curitiba: Anais da II Jornadas Latino americanas de Estudios Epistemológicos en Política Educativa.

Rodriguez, E. (2002). Cultura da juventude e da cultura escolar nas escolas secundárias do Uruguai hoje: construir uma ligação. Santiago: Última décad. v.10 n.16.

Senac (2012). Proposta Pedagógica Senac São Paulo, revitalização 2005. Projeto Ilustrativo desenvolvido para o evento Diálogos e Práticas Educacionais. São Paulo: Senac Lapa Scipião.

Silva, F.T. (2006). Cultura Escolar: quadro conceitual e possibilidades de pesquisa. Curitiba: Editora UFPR.

Silva, N.M., Freitas, V.C. (2013). Narrativas de formação como ato de reinventar-se: revelações em projetos de extensão, upe Garanhums - Brasil. In: Revista Metáfora Educacional, 2013.

$<$ www.valdeci.bio.br/revista.html $>$

Stake, R. (2011). Pesquisa qualitativa: estudando como as coisas funcionam. Porto Alegre: Peuso.

Torres, H.G., et al. (2008). Educação na periferia de São Paulo: como pensar as desigualdades educacionais? In: Ribeiro, L. C. de Q.; Kaztman, R. (Orgs.). A cidade contra a escola?: segregação urbana e desigualdades educacionais em grandes cidades da América Latina. Rio de Janeiro: Letra Capital, Faperj; Montevidéu: IPPES.

\section{Agradecimientos}

Ao Senac São Paulo, pela oportunidade de desenvolvimento profissional e académico e, sobretudo, pela contribuição com a pesquisa.

À Profa. Dra. Ecleide Cunico Furlanetto, por sua dedicação e orientação.

Aos professores do Programa de Mestrado em Educação da UNICID. 